\title{
Smart Home and Artificial Intelligence as Environment for the Implementation of New Technologies
}

\author{
Vasyl Kopytko ${ }^{1}$, Lyubov Shevchuk ${ }^{2}$, Larysa Yankovska ${ }^{2}$, Zhanna Semchuk ${ }^{2}$, \\ Rostyslav Strilchuk ${ }^{3}$
}

${ }^{1}$ Lviv Branch of Dnipropetrovsk National University of Railway Transport named after Academician V. Lazaryan

12a I. Blazhkevych Street, Lviv, 79052, Ukraine

${ }^{2}$ Lviv University of Business and Law

99 Kulparkivska Street, Lviv, 79021, Ukraine

${ }^{3}$ Lesya Ukrainka Eastern European National University

13 Voli Avenue, Lutsk, 43025, Ukraine

DOI: $10.22178 /$ pos.38-2

JEL Classification: 010

Received 01.08.2018

Accepted 15.09.2018

Published online 20.09.2018

Corresponding Author:

Zhanna Semchuk

semchuk_zv1@ukr.net

(C) 2018 The Authors. This

article is licensed under a

Creative Commons Attribution

4.0 License@ @

\begin{abstract}
The technologies of a smart home and artificial intelligence (Al) are now inextricably linked. The perception and consideration of these technologies as a single system will make it possible to significantly simplify the approach to their study, design and implementation. The introduction of $\mathrm{Al}$ in managing the infrastructure of a smart home is a process of irreversible close future at the level with personal assistants and autopilots. It is extremely important to standardize, create and follow the typical models of information gathering and device management in a smart home, which should lead in the future to create a data analysis model and decision making through the software implementation of a specialized Al. Al techniques such as multi-agent systems, neural networks, fuzzy logic will form the basis for the functioning of a smart home in the future. The problems of diversity of data and models and the absence of centralized popular team decisions in this area significantly slow down further development. A big problem is a low percentage of open source data and code in the smart home and the Al when the research results are mostly unpublished and difficult to reproduce and implement independently. The proposed ways of finding solutions to models and standards can significantly accelerate the development of specialized Als to manage a smart home and create an environment for the emergence of native innovative solutions based on analysis of data from sensors collected by monitoring systems of smart home. Particular attention should be paid to the search for resource savings and the profit from surpluses that will push for the development of these technologies and the transition from a level of prospect to technology exchange and the acquisition of benefits.
\end{abstract}

Keywords: smart home; artificial intelligence; technologies implementation.

\section{INTRODUCTION}

Smart home is a one of the most interesting things in terms of improving everyday life, opportunities, health and self-improvement. Because it is applying to each of us and directly related to IT. Smart home industry has a rapid growth now and reached 21 billion US \$ in 2017 with 53.45 billion in 2022 prediction [1]. There is no global standard for its model now but first tries with IEEE standardization (IEEE 1888.42016, IEEE SA - 2900.1, etc.), some open source and patented company's solution like Apple Homekit and others. But from the developments of the developers we can describe a model of functioning of technology Smart Home as model of hardware, network, diagnosis, management and application levels of interaction. There are many solutions now available for lower levels of interaction, which include devices for defining environmental parameters and managing of smart home. We also offer our own implementation model that includes these levels of interaction. But there is small amount of attempts to de- 
velop the latest level of interaction should be the control center of a smart home. Artificial Intelligence (AI) is a rapid growth industry too, with total size of market 2.42 billion U.S. dollars in 2017 [2]. Smart home technology is linked with $\mathrm{AI}$ and it is must be managed by this. The trends of development smart home and AI technologies now is correlated and there are already solutions in the form of intelligence advisers and informants. Understandable next step should be appearance of new kind of specialized AI that trains on specified smart home information and events. Most likely it will be protocol and software realization of neural network model decision-making process in a specific home.

Researchers such as R. Harper [3], [4], T. Hargreaves, C. Wilson [5], G. Demiris, B. K. Hensel [6], R. Moran [7], J. C. Augusto, C. D. Nugent and others were engaged in research of smart home. The artificial intelligence was investigated by R. Kurzweil [8], S. Russel, P. Norvig [9] and others. But most scientists do not consider these two concepts as a system of collaborative interaction that allows them to more effectively study the processes of their development and find practical application. The purpose of the publication is to determine smart home and artificial intelligence as combined innovative tools and environment for the implementation of new technologies.

\section{RESULTS AND DISCUSSION}

\section{Smart home and Al}

Every person can now implement technology of smart home through the availability of hardware. More devices begin to support smart functions and synchronize with each other with data accumulation. It's now even smart bracelets, toothbrushes and smartphones and not only specialized sensors and control devices. But specialized devices can be bought not only from wellknown brands but also design decisions like Arduino, Raspberry PI, etc. with lowest price and opportunities. It is possible to create your own home network with these solutions for your own needs and transform them into a distributed diagnostic, analysis and management network in their own home in the future. The main problem here is the creation and standardization of the model for future decisions, when most of the existing ones repeat the already created and the level of prevalence and the exchange of previous outputs is minimal. Existing solutions also do not allow to start even a simple implementation with the accumulation of information for future implementations. The established network of diagnostics and management should not focus primarily on monitoring functions that do not provide a practical improvement from the implementation of technology to the life of the home. The main tasks to be solved by such a network should be recommendations for improving the lives of residents of a smart home. Among them, creation of a better atmosphere (including social) in the home, optimization of processes of economy of power resources and water and selfsustainability issues. The best way to manage and train such a network is to implement a special AI. Ways of saving costs in a reasonable home are mainly from an economic point of view in the dropping the possibility of direct earnings on surplus resources at certain intervals. A smart home equipped with solar panels and windmills can use energy surplus to make money, one of such affordable solutions is now a mining process for cryptocurrency. But the issues of cryptocurrencies and mining are very new and highly controversial. This concerns both security, legality and the benefits of implementing these technologies in general. But without a doubt, cryptocurrency is also part of the process of transforming our lives with IT and a part of a new system of the future. AI is increasingly configuring now to monitor security, which is very important for home management processes. There is a similarity of the rate of money investing in cryptocurrency and AI which indicates the connection between the two events and the fact that they are elements of one system. This is evidenced by the pace of development and financing of cryptocurrencies and AI technologies, the direct link between these technologies in terms of using joint tools and implementation and their direct assignment. It should also be clear that the process of mining does not bring benefits except for the calculation of hash sequences. But the mining indirectly leads to the development of computing power and creates resources for the functioning of AI. Most modern researchers distinguish and predict three stages of development of $\mathrm{AI}$ at the present stage of development of mankind [8], [9]. The first stage is a highly specialized AI, which at this stage of development of civilization looks like a software that performs specific tasks: intelligence-chess games, text recognition, translation from one language to another, Internet search programs. The basis of the algorithms of such 
programs are neural networks with probabilistic values. Such systems are already able to conduct training by pointing out the correct result in certain cases (with the participation of a person) and self-learning in obtaining a last event that has led to a better result. Examples of this intelligence include the BlueGen chess computer that won the World Chess Championship, Google's Intelligent search and translation algorithms, Google Now, Apple's Siri and Microsoft Cortana assistants, etc. The second stage is the development of a complete or general AI, which will represent in the broad sense a versatile intelligence that is commensurate with the human, can put similar tasks from different spheres, ideas, learn from mistakes. Such intelligence is not created at this moment, although in the capabilities of the hardware, modern supercomputers from the world top 500 are approaching the computational capabilities of the human brain [8], and in the near future, even with the fall of the pace of integration of transistors in modern microprocessors, they quickly exceed them. Software development for such intelligence and brain like model, specialized artificial intelligence, decisionmaking algorithms, neural network extensions and deep learning are published in open access and can be used by programmers in during development. The third stage - super intellect, which is much smarter than human, from the best known to us. His appearance will mean the beginning of a period of technological and intellectual singularity, radical changes in society and the development of civilization. The emergence of specialized AI brings significant changes in public life, in particular in the field of social and labor relations, which will most likely lead to the following phenomena: significant reduction in occupations in which the AI will significantly outperform the human; loss of motivation in those areas where such AI will significantly outperform the human (for example, chess - competition between people is possible and preserved, but further decline of interest and transition to "amateur level" are inevitable). The continued use of robotic technologies changes the principles of the organization of financial and economic activity now, determine the new conditions of competition - both in the production and in the labor market. The consequences of the use of AI will certainly be reflected in the lives of the future person. For example, the current work of cars without a driver in the USA from Google and Apple, according to some estimates, can lead to a significant reduction in the demand for driverspeople and even result in people being banned from self-driving on most motorways. Already, most societies are not able to organize their own lives without the use of technological devices security, communications, servicing and auxiliary. Work has become an element of leisure for many peoples, replacing pets and other leisure activities. The rapid development of the field of development and implementation of AI can be compared to the pace of development of the Internet in the past, with the amendments that this development will be faster. Nowadays, the sphere of development of AI in financial investments is experiencing exponential growth (Fig. 2). AI start-up financing has increased more than 10 times between 2011 and 2017 and has reached \$ 14 billion. Total funding in 2017 was over 3 times the size of 2016 funding and over the past five years, this graph shows an increased interest in AI via the number of deals in that startup ecosystem. Whereas the rapid growth in funding occurred in 2017, the rapid growth in deals occurred in 2014 [10]. We can see dynamics of financing of AI startups and the investor interest in 2011-2017 on Figure 1.

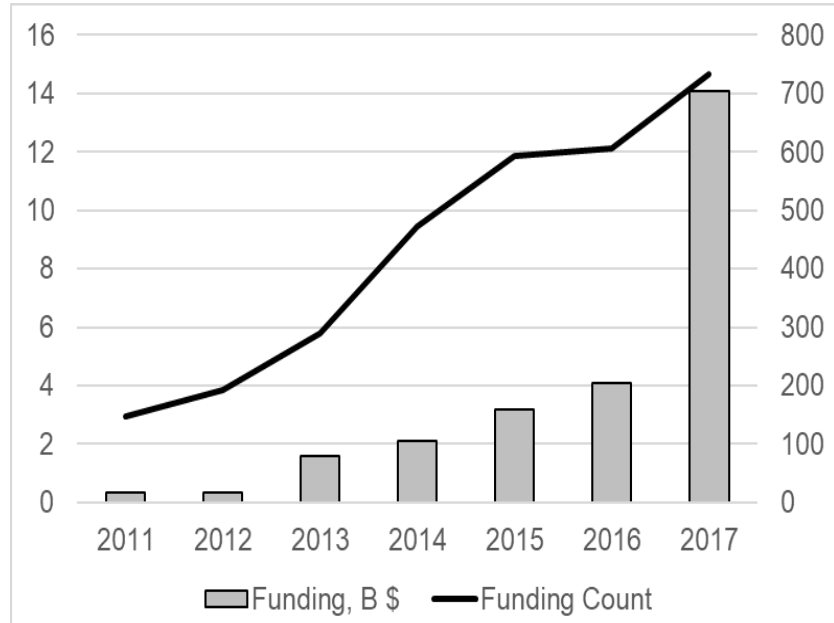

Figure 1 - Financing of artificial intelligence startups and the investor interest in 2011-2017 [10]

AI techniques such as multi-agent systems, neural networks, fuzzy logic will form the basis for the functioning of a smart home in the future. The main problems of their implementation at present are the weak dissemination of the source code for solutions and research and their copyright protection. 


\section{Smart home data collecting and decision making model}

Among the main tasks that AI should perform in a smart home will be physiological monitoring; functional monitoring and emergency detection with response; safety and security monitoring with assistance; social interaction monitoring and assistance; cognitive and sensory assistance [6]. The uniformity of sensor data and the standardization of data transfer protocols are important in this case for training neural networks to make decisions about changes in the living environment of the inhabitants of the home. A centralized intelligent decision-making body should also be able to verify the correctness of the measures being implemented. The ability to anonymously exchange information about successful decision-making is important for improving the AI of a smart home. The network of such houses will be more effective in training neural networks and getting the opportunity to exchange ready-made solutions. Existing models of data collection, analysis and decision making of a smart home are constantly being modified over time. New technologies and solutions constantly make edits that slow down standardization, even at the lowest levels. We can represent smart home data collecting and AI decision making and data exchange model on Figure 2.

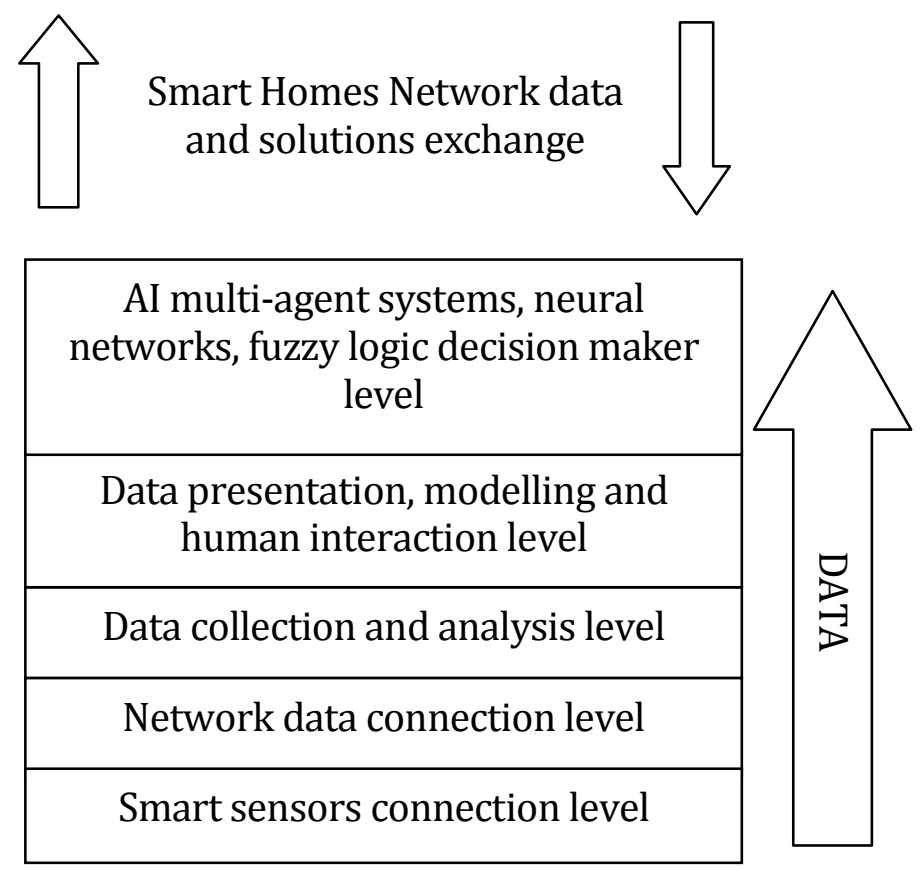

Figure 2 - Smart Home data collecting and Al decision making and data exchange model

First down level of the model is smart sensors connection level. This level has most worked out standards and smart device connection network data transmit and configuration protocols. The first problems with the same type of data are manifested in the second level of interaction network data connection level. Most existing device solutions do not have a standardized data transfer method between themselves and the central kernel of data processing. Problems are also with the transfer of teams from the center for implementation and implementation. An example of such realization is Homekit, Homebridge, MavHome etc. Data collection and analysis level is used to collect, accumulate and proc- ess data from smart devices and sensors. At the first stage, functions of this level can be assigned to personal assistants and user interaction devices. The use of a distributed system of devices is the best case for further technological implementation.

This level requires special attention in data protection and their uniformity for future use of AI. Its closely connected with data presentation, modelling and human interaction level where the user will be able to view the collected data in the form of streaming information, charts, logs and analyze the proposed solutions and their consequences. Preprocessed data in ascending order fall from level to level and finally comes in proc- 
essing to AI multi-agent systems, neural networks, fuzzy logic decision maker level. Specialized AI software and hardware in the form of NPU (neural processing units) should be used at this level. The primary task of neural networks is on base of the collected statistics predict certain events in the home. Among them, the preparation of the use of resources and devices based on the timetable of residents, load balancing and analysis of feedback. Multi agent systems must control home event triggers mechanism and neighborhoods patterns [11]. Their operation and successful work are directly related to decision trees, which should be further used as goods in the digital economy. Among the algorithms already used in software implementations of this type, ADTree, DecisionStump, LMT, MSP, RandomForest, RandomTree. But the main thing remains to create their own scenarios to improve the functioning of a smart home with AI. The implementation of scripts created by programmers in the developed software is not an expression of intelligence as such through the definition of management methods in advance. Intelligent agents must have a certain level of autonomy, on the other hand solutions must be implemented under the control of a human which significantly slows down their implementation and can be compensated by work on the network.

\section{Smart homes Network}

Smart Homes Network data and solutions exchange must determine the real economic effect and bring new opportunities creating an environment of new technological solutions. Exchange of information on the successful application of data processing by the neural networks of temperature, illumination, social interaction, etc. inside a home with a better life for the inhabitants can create demand for them and promote further development. Apparently the most valuable information will be given from neighboring houses in such a network that are located in the same climatic zone, covered by the same service providers. Possible scenarios and patterns of implementation from the network will include directions for autonomy, saving money, or improving certain living standards of the inhabitants of the home - health, activity and productivity. One of the major issues will be the security and privacy of the data that will require their anonymity and mixing. An example of implementing such solutions with the obtaining of savings or profits is also the exchange of information on energy conservation [12] or the use of its surplus to mining cryptocurrencies and saving such resources like water. The role of cryptocurrency in the information exchange network between smart homes should also become significant. One of the clear candidates for the trade and payment process in such a network is currently the IOTA crypto-coins project. Use of transaction recording systems such as Blockchain or Tangle also should significantly increase the security of the network of smart houses. The technology of functioning of an autonomous smart home in recent years without the exchange of information between different solutions cannot develop sufficiently rapidly and dynamically.

\section{CONCLUSIONS}

Therefore, the technologies of a smart home and AI are now inextricably linked. The perception and consideration of these technologies as a single system will make it possible to significantly simplify the approach to their study, design and implementation. The introduction of AI in managing the infrastructure of a smart home is a process of irreversible close future at the level with personal assistants and autopilots. It is extremely important to standardize, create and follow the typical models of information gathering and device management in a smart home, which should lead in the future to create a data analysis model and decision making through the software implementation of a specialized AI. AI techniques such as multi-agent systems, neural networks, fuzzy logic will form the basis for the functioning of a smart home in the future. The problems of diversity of data and models and the absence of centralized popular team decisions in this area significantly slow down further development. A big problem is the low percentage of open source data and code in the smart home and the AI when the research results are mostly unpublished and difficult to reproduce and implement independently. The proposed ways of finding solutions to models and standards can significantly accelerate the development of specialized AIs to manage a smart home and create an environment for the emergence of native innovative solutions based on analysis of data collected by monitoring systems. Smart Homes Network data and solutions exchange must determine the real economic effect and bring new opportunities 
creating an environment of new technological solutions. The role of cryptocurrency in the information exchange network between smart homes should also become significant. One of the clear candidates for the trade and payment process in such a network is currently the IOTA crypto project. Particular attention should be paid to the search for resource savings and the profit from surpluses that will push for the development of these technologies and the transition from a level of prospect to technology exchange and the acquisition of benefits.

\section{REFERENCES}

1. Statista. (2018). Forecast market size of the global smart home market from 2016 to 2022 (in billion U.S. dollars). Retrieved July 1, 2018, from https://www.statista.com/statistics/682204/globalsmart-home-market-size/

2. Statista. (2018). Revenues from the artificial intelligence (AI) market worldwide from 2016 to 2025 (in million U.S. dollars). Retrieved July 1, 2018, from https://www.statista.com/statistics/607716/worldwide-artificial-intelligence-marketrevenues/

3. Harper, R. (2005). Inside the Smart Home. London: Springer.

4. Harper, R. (2012). The Connected Home: The Future of Domestic Life. Dordrecht: Springer.

5. Hargreaves, T., \& Wilson, C. (2017). Smart Homes and Their Users. Cham: Springer International Publishing

6. Hensel, B. K., \& Demiris, G. (2008). Technologies for an Aging Society: A Systematic Review of "Smart Home" Applications. Yearbook of Medical Informatics, 17(01), 33-40. doi: 10.1055/s-00381638580

7. Moran, R. (1993). The electronic home: Social and spatial aspects. Luxemburg: Office for official publications of the European communities.

8. Kurzweil, R. (2016). The singularity is near: When humans transcend biology. London: Duckworth.

9. Russell, S., \& Norvig, P. (2003). Artificial Intelligence: A Modern Approach (2nd ed.). Upper Saddle River: Pearson.

10. Venture Scanner. (2018, January 19). Interest in AI Startups Exploded in 2017. Retrieved from https://www.venturescanner.com/blog/2018/interest-in-ai-startups-exploded-in-2017

11. Augusto, J. C., \& Nugent, C. D. (2006). Designing Smart Homes: The Role of Artificial Intelligence. Berlin Heidelberg: Springer-Verlag GmbH.

12. Teich, T., Roessler, F., Kretz, D., \& Franke, S. (2014). Design of a Prototype Neural Network for Smart Homes and Energy Efficiency. Procedia Engineering, 69, 603-608. doi: 10.1016/j.proeng.2014.03.032 